PAPER

\title{
Phase Noise of Multiphase CMOS LC Oscillators Coupled by Mutual Inductors
}

\author{
Tatsuya Imanishi ${ }^{1}$ and Seiichiro Moro ${ }^{2}$ \\ Faculty of Engineering, University of Fukui \\ 3-9-1 Bunkyo, Fukui 910-8507, Japan \\ E-mail: ${ }^{1}$ tatsuya.imanishi10330071@gmail.com, ${ }^{2}$ moro@u-fukui.ac.jp
}

\begin{abstract}
There have beem many studies on the analysis and design of low-noise oscillators. Recently, much attention has been paid to the noise reduction technique using coupled oscillators. When oscillators are coupled, the coupling method is very important and affects various factors, for example, the level of noise. In this study, we analyze the phase noise of multiphase CMOS LC oscillators coupled by mutual inductors by using the impulse sensitivity function. From the simulation results, using mutual inductors as coupling elements can reduce the phase noise. Also, for oscillators coupled by mutual inductors, we show that setting a coupling coefficient of around 0.2 can realize the lowest phase noise near the oscillation frequency.
\end{abstract}

Keywords: coupled oscillators, multiphase oscillations, phase noise, impulse sensitivity function

\section{Introduction}

Recently, many studies on the analysis and design of low-noise oscillators have been reported $[1,2]$. In particular, oscillators that can generate a quadrature or multiphase sine wave are useful for application to injection locked frequency multipliers (ILFMs) [3] and as local oscillators of phase-locked loop and frequency synthesizers. A high-frequency oscillator was proposed by Romano et al. as a multiphase oscillators[1]. $N$ CMOS LC oscillators in a ring topology are coupled by transistors in this circuit. Because the coupling elements are nonlinear, it is considered that the noise in the circuit will be higher than that for linear coupling elements.

From the viewpoint of coupling elements, we have proposed $N$ CMOS LC oscillators in a ring topology, which are coupled by mutual inductors[2]. Because the coupling elements are linear, the reduction of noise can be expected in comparison with the circuit shown in Ref.[1]. Also, downsizing of the circuit has been an important theme. It is considered that the effect of the coupling coefficient of the mutual inductance is important when the downsizing of the circuit is considered.

In this study, we analyze the phase noise of multiphase CMOS LC oscillators coupled by mutual in- ductors, and we investigate the effect of the coupling method and the coupling coefficient of the mutual inductance by simulation. To analyze the phase noise, we use the phase noise model proposed in Ref.[4], which introduces the impulse sensitivity function (ISF). By using the ISF, we can analyze oscillators with nonlinearity, and the phase noise can be calculated more accurately than by analysis with a linear approximation model of the oscillators. From the results, we show that lower phase noise is realized by changing the coupling elements from transistors to mutual inductors. Also, among mutual inductors, it is shown that setting a coupling coefficient of around 0.2 can realize the lowest phase noise near the oscillation frequency.

This paper is organized as follows. In Sect.2, the circuits used in this study, the equivalent model and the state equations are explained. In Sect.3, the phase noise model using the ISF is explained, and the method and the results of the simulation are shown. Finally, Sect. 4 gives the conclusion.

\section{Circuit Model}

Figure 1(a) shows the circuit of multiphase CMOS LC oscillators proposed in Ref.[1]. In this circuit, $N$ identical CMOS LC oscillators in a ring topology are 


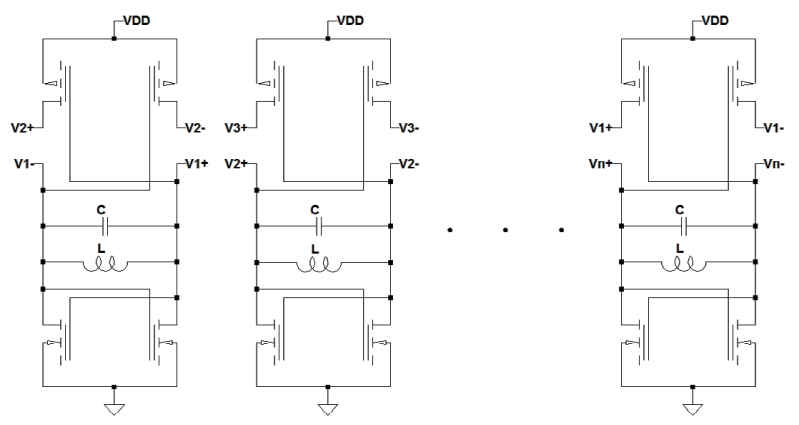

(a)

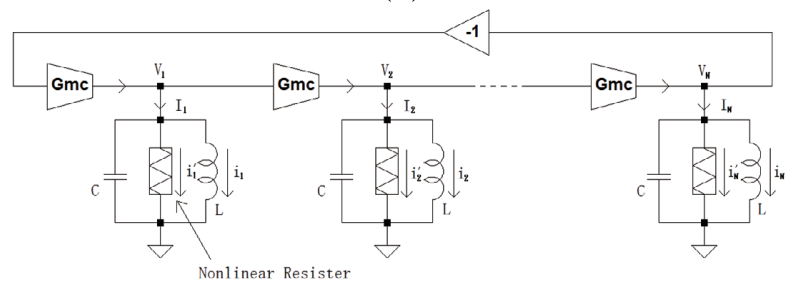

(b)

Fig. 1 Multiphase CMOS LC oscillators coupled by transistors: (a) Circuit schematic, (b) Equivalent model

coupled by CMOS transistors. Figure 1(b) shows the equivalent model of Fig.1(a). In Ref.[1], each oscillator is modeled as a linear system. However, every oscillator essentially contains nonlinearity due to the nonlinear $v-i$ characteristics of transistors in the actual circuit. Therefore, in this paper, we consider that each oscillator can be modeled as a van der Pol oscillator as shown in Ref.[5] and that the transconductance connecting the oscillators, which is composed of transistors, has nonlinear characteristics. Thus, in Fig.1, $N$ van del Pol oscillators are coupled by nonlinear transconductance $G_{m c}$, which represents the characteristics of the transistors. The characteristics of $G_{m c}$ and each nonlinear resistor in the circuit are defined respectively as follows.

$$
\begin{aligned}
I_{l} & =G_{1} V_{l-1}+G_{3} V_{l-1}^{3} \quad\left(\text { for } G_{m c}\right) \\
i_{l}^{\prime} & =-g_{1} V_{l}+g_{3} V_{l}^{3}
\end{aligned}
$$

(for each nonlinear resistor)

$$
(l=1,2, \cdots, N)
$$

To perform a simulation by numerical calculation, it is necessary to derive state equations for the oscillators. Here, we consider an equivalent model of the circuit, which is composed of $N$ van der Pol oscillators coupled in a ring topology [6].

When these $N$ oscillators are coupled as shown in Fig.1(b), the oscillators have coupling terms from the preceding oscillator, and the $l$ th oscillator satisfies the following relationship.

$$
V_{l}=L \frac{d i_{l}}{d t}
$$

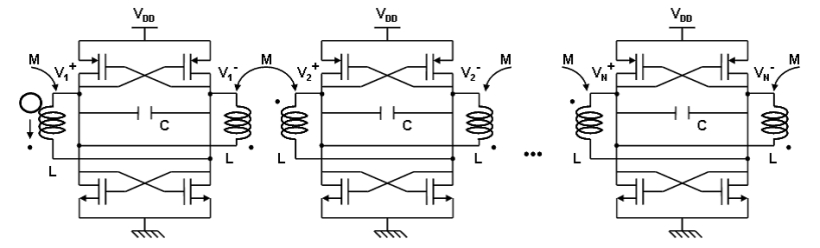

(a)

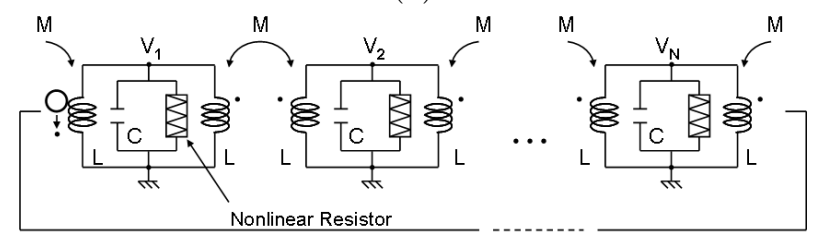

(b)

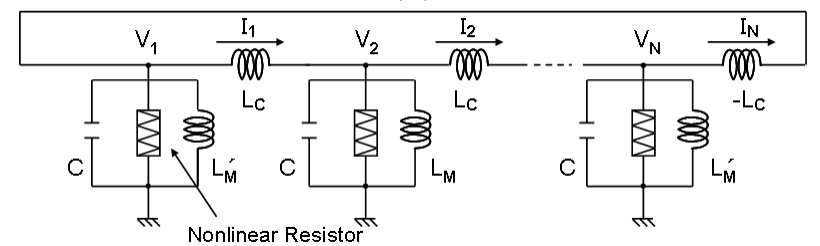

(c)

Fig. 2 Multiphase CMOS LC oscillators coupled by mutual inductors: (a) Circuit schematic, (b) Equivalent model, (c) Equivalent converted model

$$
\begin{aligned}
& I_{l}=i_{l}+C \frac{d V_{l}}{d t}+i_{l}^{\prime} \\
& (l=1,2, \cdots, N)
\end{aligned}
$$

The normalized differential equations are obtained from Eq.(2) by changing the variables as

$$
t=\sqrt{L C} \tau, \quad V_{l}=\sqrt{\frac{g_{1}}{3 g_{3}}} x_{l}, \quad i_{l}=\sqrt{\frac{g_{1} C}{3 g_{3} L}} y_{l}
$$

and by defining

$$
\varepsilon=\sqrt{\frac{L}{C}} g_{1}(\ll 1), \quad \alpha=\sqrt{\frac{L}{C}} G_{1}, \quad \beta=\sqrt{\frac{L}{C}} \frac{g_{1} G_{3}}{3 g_{3}}
$$

Then,

$$
\begin{aligned}
& \dot{y}_{l}=x_{l} \quad(l=1,2, \cdots, N) \\
& \dot{x}_{1}=-y_{1}+\varepsilon\left(x_{1}-\frac{1}{3} x_{1}^{3}\right)-\alpha x_{N}-\beta x_{N}{ }^{3} \\
& \dot{x}_{l}=-y_{l}+\varepsilon\left(x_{l}-\frac{1}{3} x_{l}{ }^{3}\right)+\alpha x_{l-1}+\beta x_{l-1}{ }^{3} \\
& (l=2,3, \cdots, N)
\end{aligned}
$$

where $\dot{x}_{l}$ and $\dot{y}_{l}$ denote $d x_{l} / d \tau$ and $d y_{l} / d \tau$, respectively.

Figure 2(a) shows the circuit of multiphase CMOS LC oscillators proposed in Ref.[2] and Fig.2(b) shows the equivalent model of Fig.2(a). In this circuit, the $N$ identical CMOS LC oscillators in a ring topology 
are coupled by mutual inductors. When Fig.2(b) is converted to Fig.2(c), the values of $L_{M}, L_{M}^{\prime}$ and $L_{C}$ are

$$
L_{M}=\frac{1+k}{2} L, \quad L_{M}^{\prime}=\frac{1-k^{2}}{2} L, \quad L_{C}=\frac{1-k^{2}}{k} L
$$

respectively, where $k=M / L$ is the coupling coefficient of mutual inductors.

When these $N$ oscillators are mutually coupled as shown in Fig.2(c), the oscillators have coupling terms from the preceding and succeeding oscillators, and the $l$ th oscillator satisfies the following relationship.

$$
\begin{aligned}
V_{l}-V_{l+1} & =L_{C} \frac{d I_{l}}{d t} \\
I_{l-1}-I_{l} & =\frac{1}{L_{M}} \int V_{l} d t+C \frac{d V_{l}}{d t}+i_{l} \\
i_{l} & =-g_{1} V_{l}+g_{3} V_{l}^{3} \\
(l & =2,3, \cdots, N)
\end{aligned}
$$

The normalized differential equations are obtained from Eq.(4) by changing the variables as

$$
t=\tau / \sqrt{\frac{1}{C L_{M}}+\frac{1}{C L_{C}}}, \quad V_{l}=\sqrt{\frac{g_{1}}{3 g_{3}}} x_{l}
$$

and by defining

$$
\begin{aligned}
\varepsilon & =g_{1} \sqrt{\frac{L_{M}(1-\alpha)}{C}}(\ll 1), \quad \alpha=\frac{L_{M}}{L_{C}+L_{M}}, \\
\alpha^{\prime} & =\frac{L_{M}^{\prime}}{-L_{C}+L_{M}^{\prime}}=-\alpha
\end{aligned}
$$

Then,

$$
\begin{aligned}
\dot{x}_{l} & =y_{l} \quad(l=1,2, \cdots, N) \\
\dot{y}_{1} & =\varepsilon\left(1-x_{1}^{2}\right) y_{1}-(1+\alpha) x_{1}+\alpha^{\prime}\left(x_{N}-x_{2}\right) \\
\dot{y}_{l} & =\varepsilon\left(1-x_{l}^{2}\right) y_{l}-(1+\alpha) x_{l}+\alpha\left(x_{l-1}+x_{l+1}\right) \\
(l & =2,3, \cdots, N-1) \\
\dot{y}_{N} & =\varepsilon\left(1-x_{N}^{2}\right) y_{N}-(1+\alpha) x_{N}+\alpha^{\prime}\left(-x_{N-1}+x_{1}\right)
\end{aligned}
$$

where $\dot{x}_{l}$ denotes $d x_{l} / d \tau$, i.e., $y_{l}$ is the slope of $x_{l}$ with respect to the normalized time $\tau$. In Eq.(5), $\varepsilon$ is the strength of the nonlinearity and $\alpha$ and $\alpha^{\prime}=-\alpha$ are the coupling factors. Although $\varepsilon$ depends on the value of $\alpha$, it is considered that the effect of the variation of $\varepsilon$ accompanying the variation of $\alpha$ should be small because we assume $\varepsilon \ll 1$.

Equations (3) and (5) will be used for analyses of the phase noise of the oscillators in the next section.

\section{Simulation}

\subsection{Phase noise model using ISF}

In this section, we calculate the phase noises of the multiphase oscillators shown in Sect.2 by using the
ISF proposed in Ref.[4]. Usually, when we analyze the phase noise of oscillators, linearized models of the oscillators are used. However, as the oscillators must essentially include nonlinear elements, the phase noise analyzed by linear models is not accurate since the effect of nonlinearity is ignored. By using the ISF, we can analyze the oscillators with nonlinearity, and the phase noise can be calculated more accurately.

Equations (6) and (7) give the phase noise model in the $1 / f^{2}$ region and $1 / f^{3}$ region, respectively, and Eq.(8) represents the $1 / f^{3}$ phase noise corner, as shown in Ref.[4].

$$
\begin{aligned}
L(\Delta \omega) & =10 \log \left(\frac{\overline{i_{n}^{2}} \Gamma_{r m s}^{2}}{4 q^{2} \Delta \omega^{2}}\right) \\
L(\Delta \omega) & =10 \log \left(\frac{c_{0}^{2} \overline{i_{n}^{2}} \omega_{1 / f}}{8 q^{2} \Delta \omega^{3}}\right) \\
\omega_{1 / f^{3}} & =\omega_{1 / f} \cdot \frac{c_{o}^{2}}{2 \Gamma_{r m s}^{2}}
\end{aligned}
$$

Here, $\Delta \omega$ is the offset frequency, $\overline{i_{n}{ }^{2}}$ is the white power spectral density, $q$ is the maximum charge displacement across the capacitor on the output node, $\Gamma_{r m s}$ is the RMS of the ISF, $c_{0}$ is the DC component of the Fourier coefficient of the ISF, and $\omega_{1 / f}$ is the corner frequency of the device $1 / f$ noise.

Figure 3 shows schematic diagrams of the oscillator's noise. The phase noises in the $1 / f^{2}$ region and $1 / f^{3}$ region are due to white noise and $1 / f$ noise, respectively. From Eq.(8), the $1 / f^{3}$ phase noise corner is smaller than the $1 / f$ device noise corner by a factor of $c_{0}^{2} / 2 \Gamma_{r m s}^{2}$. From Eqs.(7) and (8), the phase noise near the oscillation frequency depends on $c_{0}$. Therefore, it is important to reduce the DC component of the ISF.

The ISF is derived to obtain $\Gamma_{r m s}^{2}$ and $c_{0}^{2}$ by calculating Eqs.(3) and (5) and using their impulse responses. We use the fourth-order Runge-Kutta method for the numerical calculation. The procedure to obtain the ISF is as follows.

(A) An impulse is injected to the voltage, which is in a steady state, and the time at the peak point is measured when the voltage reaches a steady state again.

(B) A time gap $\Delta t$ is derived by calculating the difference between the time measured in (A) and the time at the case that the impulse is not injected in $(\mathrm{A})$.

(C) (A) and (B) are repeated by changing the point of injection continuously until it has moved one cycle of the voltage waveform.

This procedure is shown in Fig. 4. The amplitude and pulse width of the impulse are set to 1 and 0.001 , respectively. 


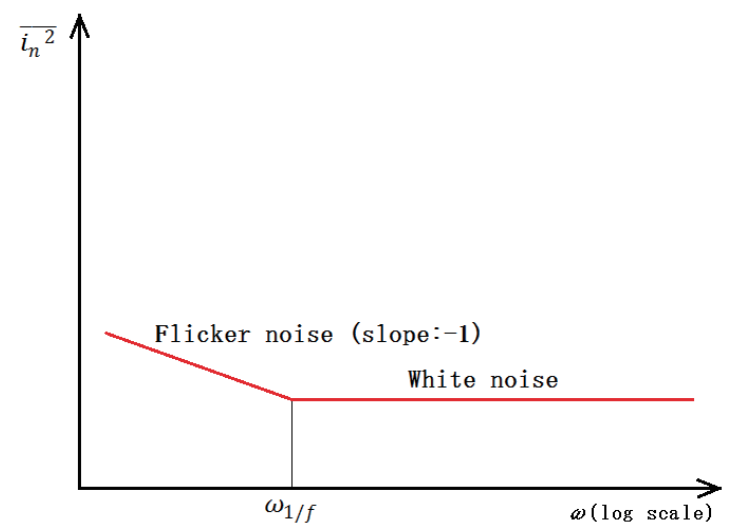

(a)

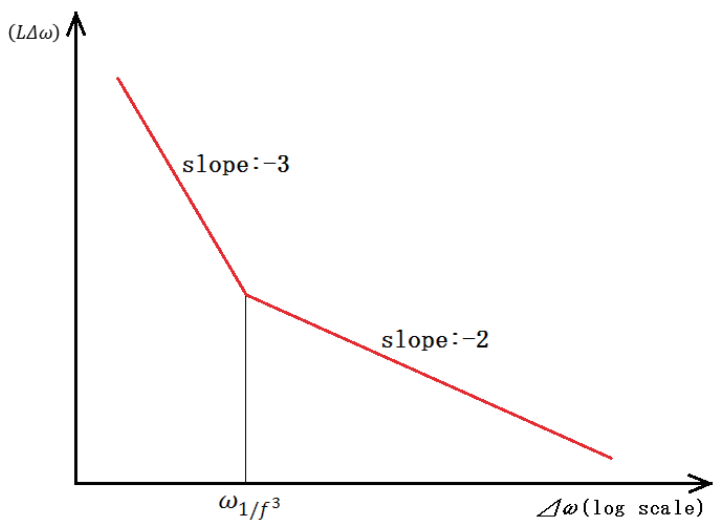

(b)

Fig. 3 (a) Internal noise, (b) Phase noise

The waveforms of the ISF, which are derived from the circuits coupled by transistors and by mutual inductors, are shown for $N=3-6$ in Fig.5. The amplitude is normalized by the maximum amplitude in Fig.5(a) for $N=3$. The coupling coefficient of the mutual inductance is set as 0.3 , which corresponds to $\alpha=0.176$, and the strength of the nonlinearity $\varepsilon$ is set as 0.01 . $\Gamma_{r m s}^{2}$ is derived by Parseval's relation as follows:

$$
\sum_{n=0}^{\infty} c_{n}^{2}=\frac{1}{\pi} \int_{0}^{2 \pi}|\Gamma(x)|^{2} d x=2 \Gamma_{r m s}^{2}
$$

where $c_{n}$ is a Fourier coefficient and $\Gamma(x)$ is the ISF. In the calculation of $\Gamma_{r m s}^{2}$, the partial sum from $c_{0}$ to

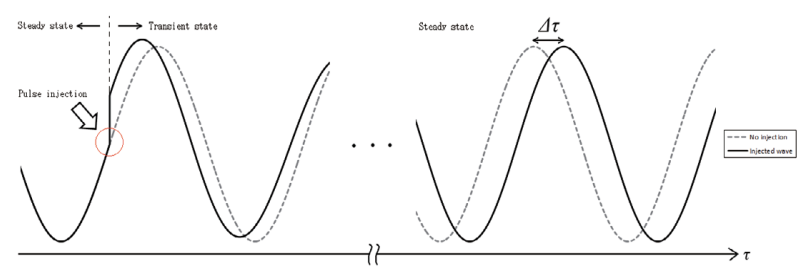

Fig. 4 Calculation process of ISF

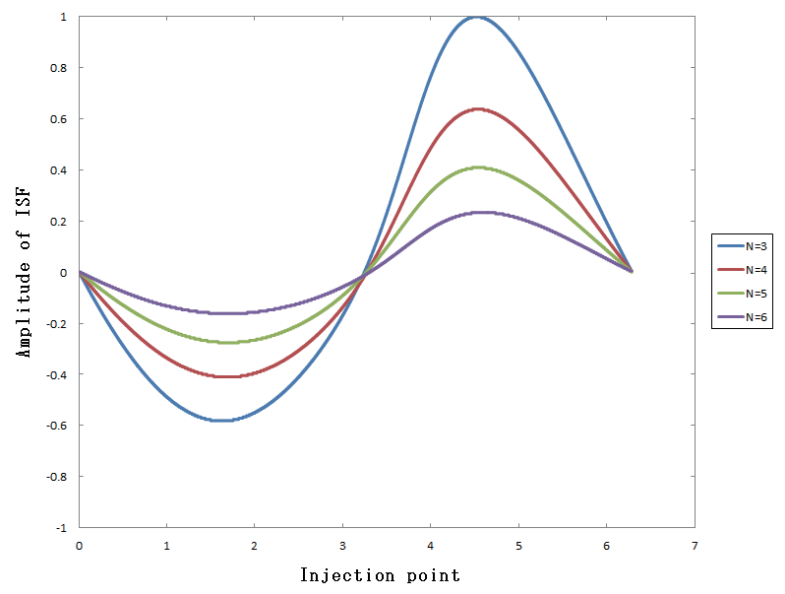

(a)

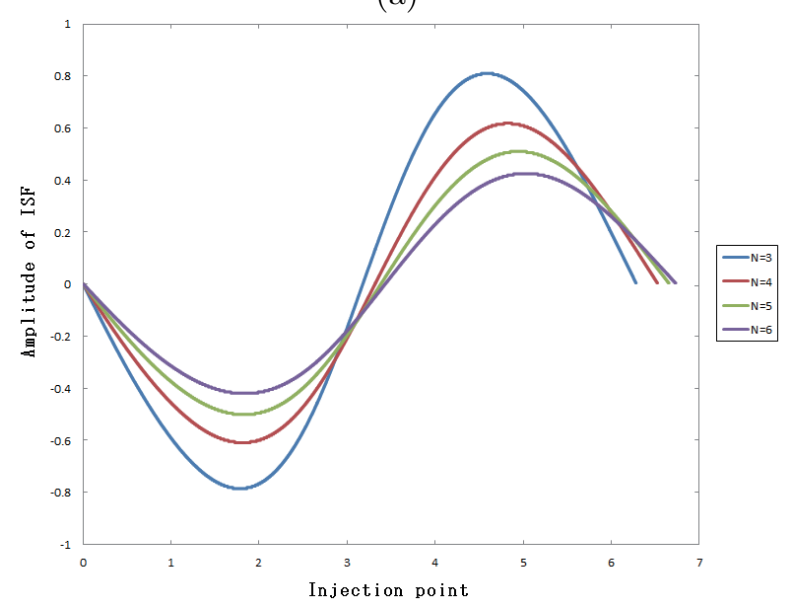

(b)

Fig. 5 Waveforms of ISF: (a) Coupled by transistors, (b) Coupled by mutual inductors

$c_{10}$ is used.

3.2 Phase noise comparison with different coupling methods

The calculated $\Gamma_{r m s}^{2}$ and $c_{0}^{2}$ are shown in Fig.6. Moreover, we show the effect of the coupling methods on the phase noise by difference of only Fourier coefficient of the ISF in Fig.7 for $N=3$. In Fig. $7, \overline{i_{n}{ }^{2}}$, $q$ and $\omega_{1 / f}$ are assumed to have the same values for the two kinds of coupling method. Also, it is assumed that $\omega_{1 / f} / 2 \pi=500 \mathrm{kHz}$.

The $1 / f^{3}$ phase noise corner is calculated as $\omega_{1 / f^{3}}=0.01 \mathrm{~Hz}$ for the oscillators coupled by mutual inductors. Therefore, in this circuit, the phase noise is expressed using only the $1 / f^{2}$ region. On the other hand, the $1 / f^{3}$ phase noise corner is calculated as $\omega_{1 / f^{3}}=6137.75 \mathrm{~Hz}$ for the oscillators coupled by transistors. From this, the region of the $1 / f^{3}$ phase noise is broad, and therefore the phase noise near the oscillation frequency is worse in the oscillators cou- 


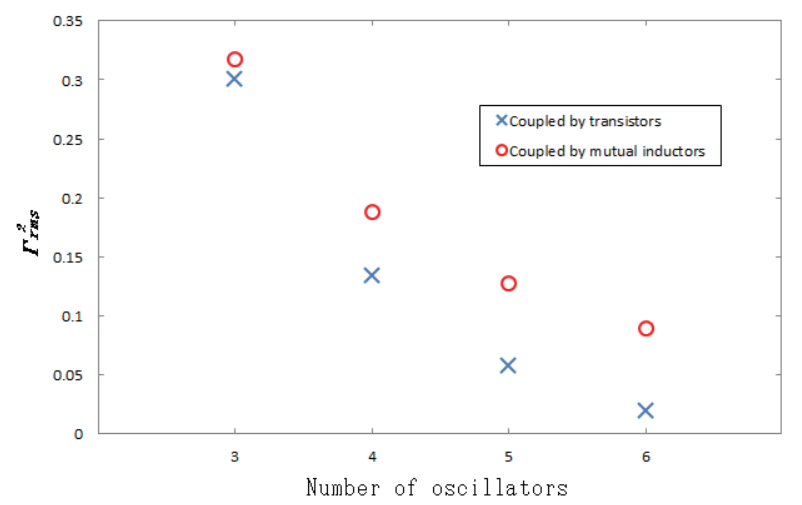

(a)

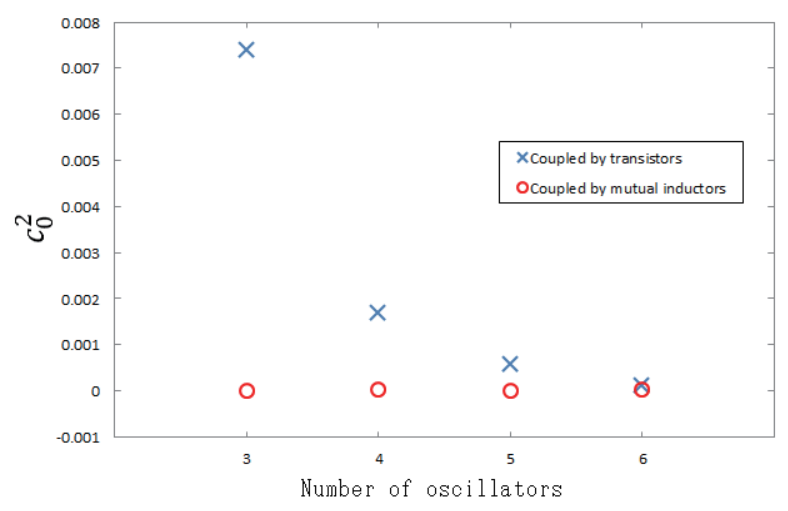

(b)

Fig. 6 Calculated (a) $\gamma_{r m s}^{2}$ and (b) $c_{0}^{2}$

pled by transistors. Similar results are obtained for different $N$.

Multiphase oscillators can realize high frequency resolution[3]. However, to realize high frequency resolution, low phase noise is needed. Therefore, the oscillators coupled by mutual inductors are more useful for a frequency synthesizer than the oscillators coupled by transistors as we predicted in Ref.[2].

\subsection{Effect of coupling coefficients}

Similarly, we examine the effect of a change in the value of the coupling coefficient $k$. The waveforms of the ISF derived from the oscillators coupled by mutual inductors for $N=3-6$ are shown in Fig.8. The coupling coefficient $k$ is varied from 0.1 to 0.9 . The amplitude is normalized by the maximum amplitude in Fig.8(a) when $k=0.1$.

The ISF expresses the sensitivity for the impulse at the injection point. In Fig.8(a), both the negative peak point and positive peak point are largest for $k=0.1$. Thus, the top and bottom asymmetry characteristics of waveforms are varied by changing the value of $k$. From Figs.8(b)-(d), the period of the waveform becomes longer by increasing the value of $k$. This characteristic becomes more obvious as the num-

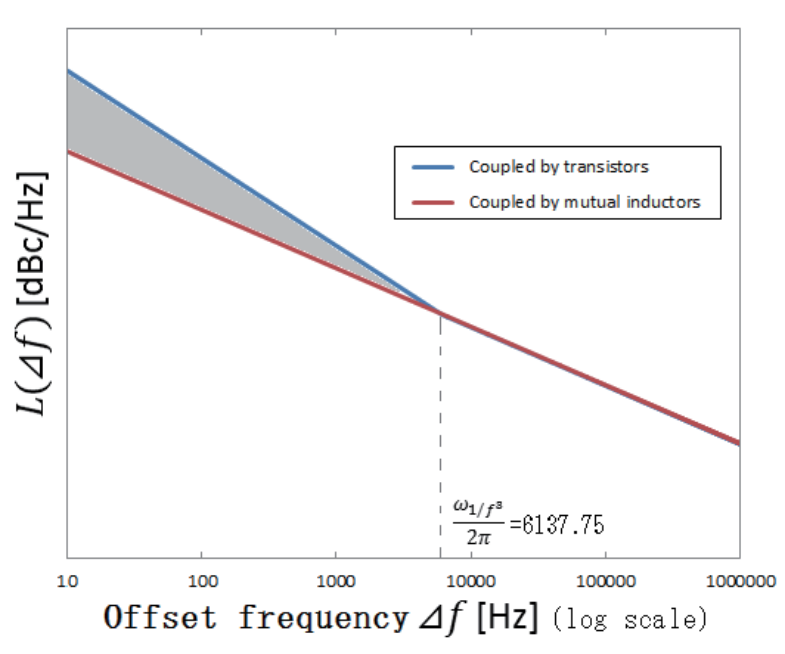

Fig. 7 Comparison of the phase noise for $N=3$

ber of oscillators is increased. From Figs.8(a)-(d), the amplitude is decreased by increasing the number of oscillators $N$.

Figure 9 shows the values of $c_{0}^{2}$ and $\Gamma_{r m s}^{2}$ for different values of $k$, derived from the waveforms of Fig. 8 and Eq.(9). There is little difference in $\Gamma_{r m s}^{2}$ due to a change in $k$ for $N=3$ (From Eq.(6), the maximum difference in the phase noise in the $1 / f^{2}$ region due to a change in $\Gamma_{r m s}^{2}$ is $0.0086 \mathrm{~dB}$ ). When $N=4-6$, $\Gamma_{r m s}^{2}$ increases with $k$. It can also be seen that $c_{0}^{2}$ is almost zero for $k=0.2$ for each number of oscillators $N$. This means that the phase noise near the oscillation frequency is reduced because of the reduction of the $1 / f^{3}$ phase noise corner.

From Eqs.(6)-(8) and the above points, it is considered that setting $k=0.2$ is the best because the phase noise in the $1 / f^{3}$ region is smallest, and the phase noise in the $1 / f^{2}$ region is relatively small for each $N$. This value of $k$ is reasonable for the design of mutual inductors with spiral inductors on VLSI chip. Therefore, we can propose a low-noise multiphase oscillator that is suitable for VLSI implementation.

When we consider the implementation in real circuits, the mismatch of the circuit parameters should be taken into account. In this case, the optimal value of $k$ can be changed. However, it is considered that the analyses in this paper can be applied to circuits with a parameter mismatch. Precise analyses are a future problem.

\section{Conclusions}

In this paper, we have investigated the advantage of the coupling method and the effect of the coupling coefficient of multiphase CMOS LC oscillators coupled by mutual inductors on phase noise.

From simulation results obtained by analyses using 


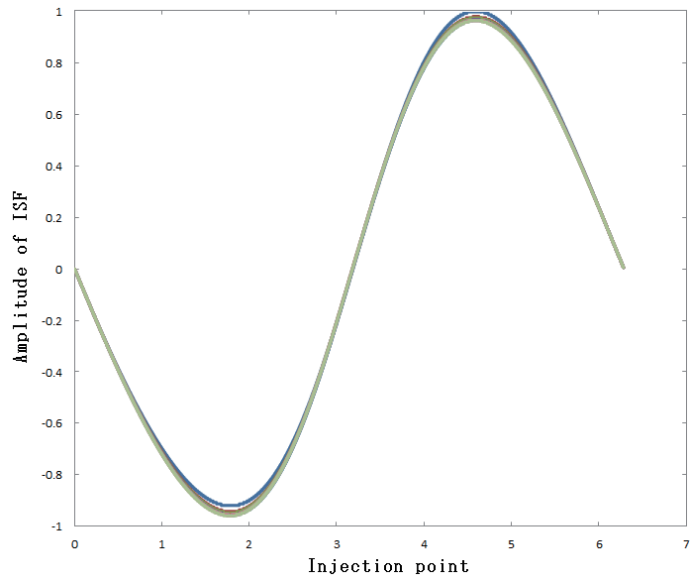

(a)

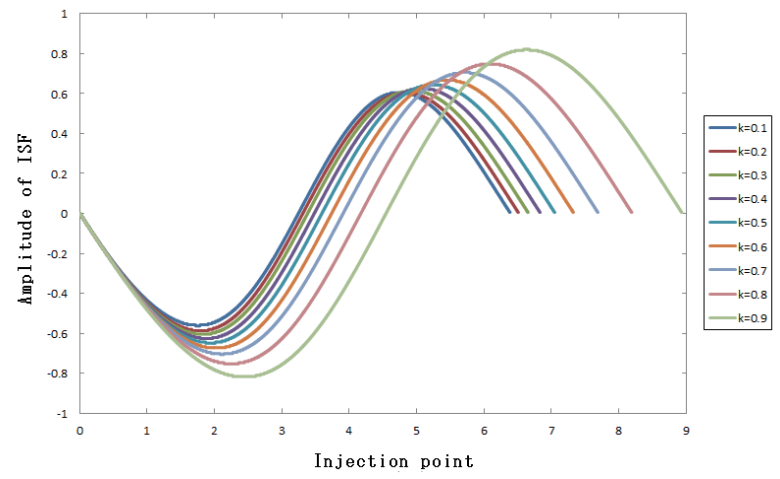

(c)

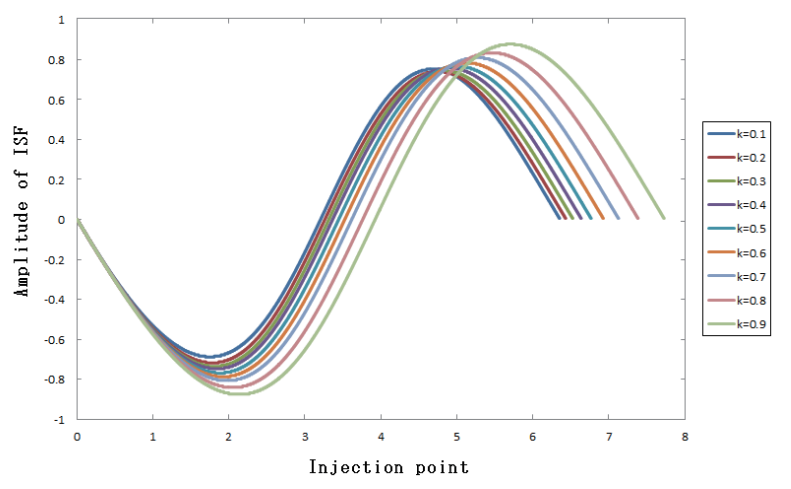

(b)

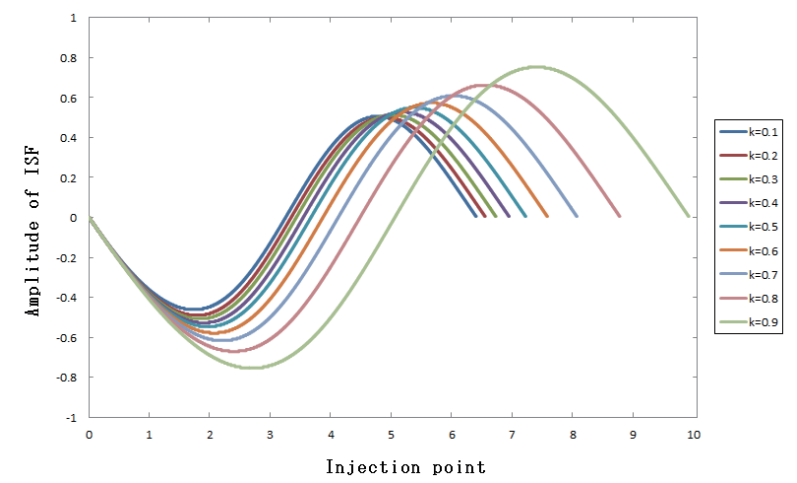

(d)

Fig. 8 Waveforms of ISF: (a) $N=3$, (b) $N=4$, (c) $N=5$, (d) $N=6$

the impulse sensitivity function (ISF), the improvement of the phase noise in the $1 / f^{3}$ region can be seen by changing coupling elements from transistors to mutual inductors as expected.

Also, we have investigated the effect of the coupling coefficient on the phase noise of multiphase CMOS LC oscillators coupled by mutual inductors. From simulation results, it is considered that setting the coupling coefficient to around 0.2 can realize low phase noise near the oscillation frequency in oscillators coupled by mutual inductors. We expect that this circuit will be useful for low-noise multiphase oscillators and for application to frequency synthesizers.

By changing the coupling elements, the number of oscillators or the coupling coefficient, it is considered that the magnitude of internal noise can be varied. Therefore, it is necessary to compare the phase noise concretely in future work. The analyses of cases that each oscillator has some parameter mismatch are also a future problem.

\section{References}

[1] L. Romano, S. Levantino, C. Samori and A. L. Lacaita: Multiphase $L C$ oscillators, IEEE Trans. Circuits Syst.-I, Vol.53, No.7, pp.1579-1588, 2006.

[2] T. Kawanari and S. Moro: Multi-phase oscillation in RF CMOS LC oscillators coupled by mutual inductors, J. Circuits Syst. Comput., Vol.19, No.4, pp.733-747, 2010.

[3] S. Ikeda, S.-Y. Lee, T. Kamimura, H. Ito, N. Ishihara and K. Masu: Fractionally injection-locked frequency multiplication technique with multi-phase ring voltage-controlled oscillator, Jpn. J. Appl. Phys., Vol.52, 04CE15, 2013.

[4] A. Hajimiri and T. H. Lee: A general theory of phase noise in electrical oscillators, Proc. IEEE, Vol.33, No.2, pp.179194, 1998

[5] S. K. Magierowski and S. Zukotynski: CMOS $L C$-oscillator phase-noise analysis using nonlinear models, IEEE Trans. Circuits Syst.-I, Vol.51, No.4, pp.664-677, 2004.

[6] T. Endo and S. Mori: Mode analysis of a ring of a large number of mutually coupled van der Pol oscillator, IEEE Trans. Circuits Syst., Vol.CAS-25, No.1, pp.7-18, 1978. 


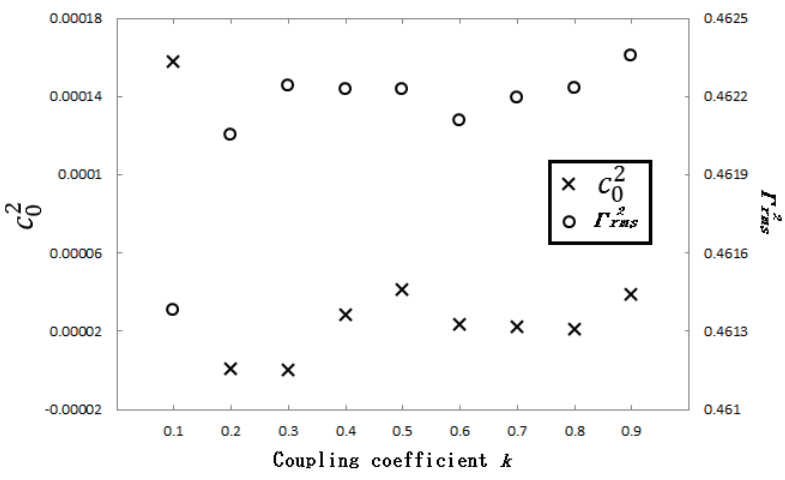

(a)

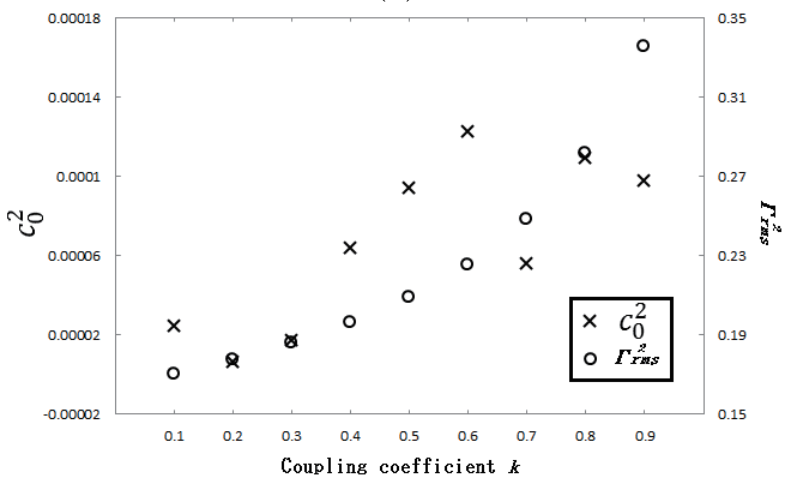

(c)

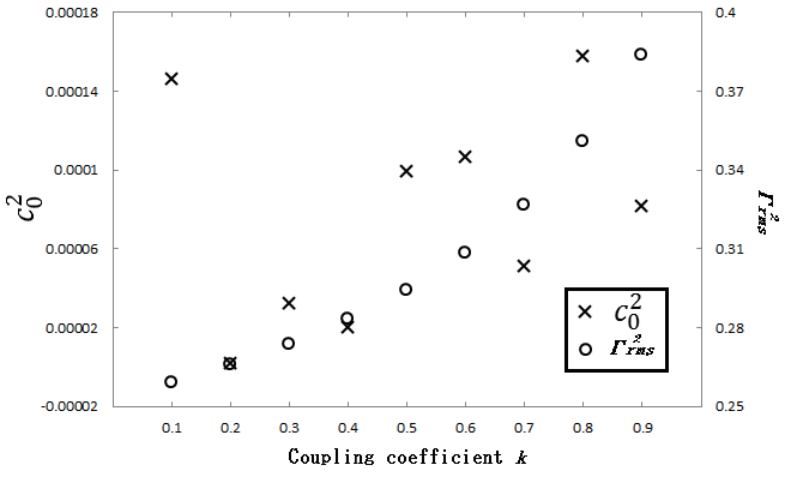

(b)

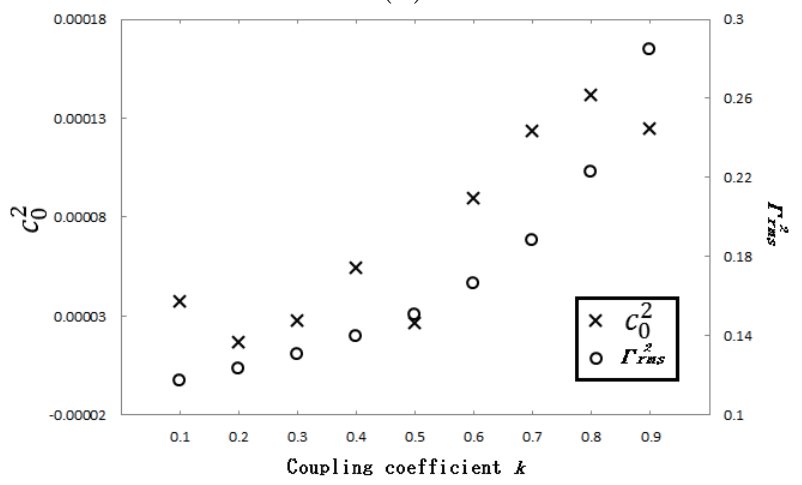

(d)

Fig. 9 Values of $c_{o}^{2}$ and $\Gamma_{r m s}^{2}$ : (a) $N=3$, (b) $N=4$, (c) $N=5$, (d) $N=6$

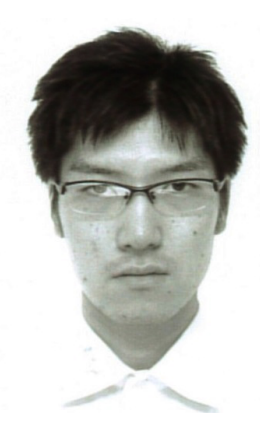

Tatsuya Imanishi received his B.E. and M.E. degrees in electrical and electronics engineering from University of Fukui, Fukui, Japan, in 2014 and 2016, respectively. His research interests are in speech signal processing and synchronization phenomena in coupled oscillators. He is now with NTC Corporation, Japan.

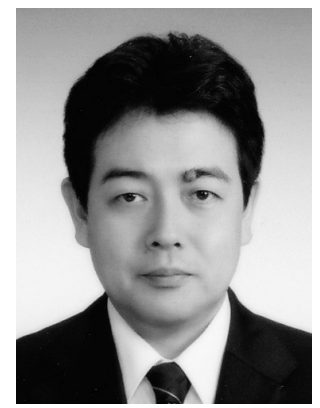

Seiichiro Moro received his B.E., M.E., and Ph.D degrees in electrical engineering from Keio University, Yokohama, Japan, in 1993 , 1995, and 1998, respectively. From 1995 to 1998 , he was a research fellow of the Japan Society for the Promotion of Science. In 1998, he joined the Department of Electrical and Electronics Engineering, University of Fukui, where he is an Associate Professor. His research interests are in nonlinear circuits, neural networks, and wavelet analysis. Dr. Moro is a member of the Institute of Electronics, Information and Communication Engineers (IEICE), the Institute of Electrical Engineers of Japan (IEEJ), the Society of Instrument and Control Engineers (SICE) and the Institute of Electrical and Electronics Engineers (IEEE).

(Received May 16, 2016; revised July 19, 2016) 